\title{
The effects of recall-concurrent visual-motor distraction on picture and word recall
}

\author{
MICHAEL W. WARREN \\ Randolph-Macon College, Ashland, Virginia 23005
}

\begin{abstract}
The dual-coding model (Paivio, 1971, 1975) predicts a larger imaginal component in the recall of pictures relative to words and a larger imaginal component in the recall of concrete words relative to abstract words. These predictions were tested by examining the effect of a recall-concurrent imagery-suppression task (pursuit-rotor tracking) on the recall of pictures vs picture labels and on the recall of concrete words vs abstract words. The results showed that recall-concurrent pursuit-rotor tracking interfered with picture recall, but not word recall (Experiments 1 and 2); however, there was no evidence of an effect of recall-concurrent tracking on the recall of concrete words (Experiment 3). The results suggested a revision of the dual-coding model.
\end{abstract}

It is now well established that the verbal recall of simple pictures is superior to the verbal recall of picture labels (e.g., Lieberman \& Culpepper, 1965; Paivio \& Csapo, 1969, 1973; Paivio, Rogers, \& Smythe, 1968; Sampson, 1970; Scott, 1967). Indeed, the picturesuperiority effect is robust. The effect is observed with pure- and mixed-list presentation (e.g., Paivio \& Csapo, 1973; Sampson, 1970) and for intentional and incidental instructions (e.g., Cohen, 1973; Paivio \& Csapo, 1973; Sampson, 1970). Likewise, pictures are better recalled when picture and word presentation is accompanied by a concurrent task designed to restrict rehearsal (Cohen, 1973) and when recall is delayed by an interpolated auditory distractor task (Pellegrino, Siegel, \& Dhawan, 1975, 1976).

Two general hypotheses have been offered to account for the picture-superiority effect. They may be broadly labeled the uniprocess hypothesis and the dual-coding hypothesis. While the uniprocess hypothesis assumes several forms, all of the forms are consistent in suggesting that both pictures and words are encoded in an equivalent manner, but that pictures are, for various reasons, "better" encoded, and thereby more likely to be recalled. In contrast, Paivio's $(1971,1975)$ dualcoding hypothesis includes the assertion that pictures and words are encoded via dual imaginal and verbal codes, but that the likelihood of dual coding is higher for pictures, thereby producing the picture-superiority effect.

One form of the uniprocess hypothesis asserts that, while both pictures and words are encoded in the same manner, the physical characteristics of pictures, such as vividness or multiplicity of cues, enhance picture

This paper is based on a dissertation-submitted to Vanderbilt University in partial fulfillment of the requirements of the $\mathrm{PhD}$ degree. I am indebted to my advisor, Keith Clayton, and Terry Allen, John Bransford, Emily Davidson, and Jeffrey Franks for criticism during the conduct of this research. recall relative to word recall. To be more specific, one might suggest that pictures afford more retrieval cues with which to query memory, thus increasing the likelihood of retrieval. Bousfield, Esterson, and Whitmarsh (1957) offered a multiplicity-of-cues explanation to account for their observation that recall was highest for labels presented with corresponding colored pictures, next for labels with corresponding uncolored pictures, and lowest for labels alone. On the other hand, Paivio, Rogers, and Smythe (1968) failed to detect any significant differences in recall for colored pictures and black-and-white pictures. Similarly, Nelson, Metzler, and Reed (1974) tested the hypothesis that the superior recognition of pictures over matching verbal descriptions was due to pictorial details, absent in the descriptions, providing the basis for differentiating target pictures from distractors, thereby facilitating recognition. However, Nelson et al. failed to detect differences between photographs and unembellished line drawings of the main theme of the photographs for either immediate or delayed recognition performance. Thus, recent research provides very little evidence to support a multiplicity-of-cues explanation for the differential memorability of pictures and words.

Davies, Milne, and Glennie (1973) have proposed the active-encoding hypothesis. They have suggested that the presentation of a picture prompts the active retrieval and generation of the appropriate label, but that a printed label may be passively read with a minimum of cognitive involvement. Thus, the activeencoding hypothesis provides a rationale for "better" verbal coding of labels with pictorial presentation. On this basis, Davies et al. predicted that viewing the name of an object just prior to viewing its pictorial referent should prompt poorer retention than the converse. The results of two experiments were consistent with the active-encoding hypothesis. An experiment by Madigan (1974) yielded an equivalent result. Thus, the active-encoding hypothesis is not an implausible account of the picture-superiority effect. 
A third form of the uniprocess hypothesis is based on Craik and Lockhart's (1972) levels-of-processing model. In this model, it is assumed that pictures are processed to a deeper level than are words, where deeper levels of processing are associated with more elaborate, more enduring, stronger traces and better recall. Recent research on semantic-decision latency suggests a rationale for the proposed difference in depth of processing, Pellegrino, Rosinski, and Siegel (Note 1) observed that subjects were significantly faster at samedifferent semantic category judgments for picture pairs than for word pairs. The result suggested that pictures enjoy more rapid access to semantic levels, thereby providing more time for semantic processing.

The alternative to uniprocess explanations of superior picture memorability is Paivio's (1971, 1975) dualcoding model. The dual-coding model asserts that pictures and words are coded via qualitatively different imaginal and verbal codes, and that the relative availability of the codes produces the picture-superiority effect. The ranking of code availability is as follows: imaginal coding of pictures, verbal coding of words, verbal coding of pictures, and imaginal coding of words. The summative availability of both codes is higher for pictures. The code redundancy increases the probability of recalling pictures relative to words, and accounts for the picture-superiority effect.

Consider some evidence for the dual-coding model. Paivio and Csapo (1969) have demonstrated that a presentation rate designed to eliminate the labeling of pictures, but not the reading of words, eliminated the picture-superiority effect for free recall. Presumably, the rapid presentation rate reduced the availability of the verbal code to pictures. Likewise, Paivio and Csapo (1973) report that orienting instructions that required subjects to draw or to imagine drawing the referents of words in an incidental paradigm produced free recall performance equivalent to that of subjects instructed to write or pronounce the labels of pictures. Presumably, the draw and imagine-drawing instructions increased the likelihood of coding words imaginally. Independent research has provided further support for the dualcoding hypothesis. Wells (1973) reports substantial picture-word and word-picture release from proactive interference, thereby suggesting differential coding of pictures and words. Wicker and Holley (1971) report that visual distraction has a larger interfering effect with pictorial than with verbal stimuli in a paired associate learning paradigm. Finally, Pellegrino et al. $(1975,1976)$ have provided results suggesting that the short-term retention of pictures is mediated by dual visual and verbal short-term stores. They suggest that, if pictures were afforded dual visual and verbal short-term representation, the pictures should be differentially susceptible to dual auditory-visual interpolated distraction. Recall in a Brown-Peterson task disclosed that picture recall was superior to word recall for auditory distraction, while the reverse obtained for dual auditory-visual distraction. Thus, the suggestion that pictures were afforded dual short-term representation received support.

The present paper reports three experiments which provide further evidence that pictures and words are represented by qualitatively different memory codes. The technique adopted was to selectively interfere with the recall of pictures by requiring subjects to engage in a visual-motor distractor task (pursuit-rotor tracking) concurrent with the free oral recall of supraspan lists. Experiment 1 demonstrates that recallconcurrent pursuit-rotor tracking interferes with the oral recall of pictures, but not of picture labels. Experiment 2 addresses explanations for the effect. Experiment 3 examines the effect of recall-concurrent pursuit-rotor tracking on the recall of concrete and abstract word lists.

\section{EXPERIMENT 1}

Recent research by Byrne (1974) and May and Clay ton (1973) suggests that conscious imaginal processes may be involved in recall. For instance, May and Clayton (1973) report that information about the appearance of an object can be remembered before the name of the object when subjects are placed in a tip-of-the-tongue state. Byrne (1974) reports a related result. He found that categorizing (e.g., animal vs nonanimal) memorized items presented as a list of words was unaffected by response mode (vocal or visually guided), while categorizing the identical items presented as a pictorial scene was significantly slower for visually guided responding. In addition, subjects presented scenes reported that they categorized the items by referring to a mental image of the scene, and that the requirement to make visually guided responses disrupted their imagery. Thus, recent research suggests that imaginal processes may mediate the recall of words and pictures. Given the dual-coding model, one would expect a larger imaginal component in the recall of pictures. That is, the increased availability of the imaginal code to pictures implies a larger imaginal component in picture recall.

Since Segal and Fusella (1970) and Brooks (Note 2) have provided results suggesting that imaginal processes and visual distractor tasks compete for the same limitedcapacity system, it seemed likely that imaginal processes involved in recall could be disrupted by engaging subjects in a recall-concurrent visual distractor task. Pursuit-rotor tracking was selected as the distractor task because Baddeley, Grant, Wight, and Thomson (1974) recently reported that pursuit-rotor tracking disrupted an imaginal memory-span task, but not a similar verbal task. Consequently, subjects were presented pictures and words and required to recall or recall while tracking. An interaction of presentation mode and recall activity was anticipated. 
Experiment 1 reports two studies. Study 1 was a preliminary study intended to test the feasibility of interfering with picture recall while only minimally interfering with word recall. Study 2 was a replication that was performed several months later, after the completion of Experiment 2.

\section{Method}

Subjects. The subjects were 32 Vanderbilt University undergraduates participating to fulfill a requirement for introductory psychology. Sixteen subjects served in each replication. Seven subjects were replaced due to equipment failure and failure to follow instructions.

Design and procedure. The design was a mixed factorial with two between-subjects and two within-subjects factors. The between-subjects factors were replications (Studies 1 and 2) and order of conditions. The within-subjects factors were presentation mode (pictures, $P$, vs words, W) and recall activity (control, C, vs tracking, T).

Subjects received one list in each of four conditions. The order of conditions (WC, WT, PC, PT; WT, PC, PT, WC; PC, PT, WC, WT; PT, WC, WT, PC) and the order of lists $(1,2,3,4$; $2,3,4,1 ; 3,4,1,2 ; 4,1,2,3)$ were counterbalanced via Latin square. The four orders of conditions were mapped on the four orders of lists, yielding 16 order-of-conditions by order-of-lists combinations. Each list appeared in each condition and ordinal position equally often. The 16 subjects in each replication were randomly assigned to the 16 order-of-conditions by order-of-lists combinations. Serial position was counterbalanced via a Latin square ordering of 4-item segments, so that each quarter of a 16-item list appeared in each of four positions for each condition. Thus, subjects receiving the same list in the same condition were presented a different order of items.

Study 1 subjects were informed that the experiment was intended to test their ability to recall pictures and words while performing and not performing a visual-motor task. Study 2 subjects were informed that the experiment was intended to study the relationship between memory for pictures and words and motor performance. Two kinds of trials were defined: recall trials (list terminated by " $R$ " in Study 1 or by "RECALL" in Study 2) and track trials (list terminated by "T" in Study 1 or by "TRACK" in Study 2). On recall trials, subjects tracked the target just long enough to touch it once and recalled for the remainder of the recall period. On track trials, subjects tracked the target for the entire recall period and recalled as they tracked. Subjects were instructed to insure that they touched the target before recalling. The instructions emphasized that recall and tracking performance were equally important on track trials. Subjects received oral, free recall instructions and were informed that their recall protocols would be tape recorded. Study 2 subjects were encouraged to guess and were instructed not to be afraid to repeat items. Practice consisted of familiarization with the tracking task (approximately 5-10 sec of tracking) and two practice trials, a word-recall and a picturetrack trial on the same 12-item list. Subjects were informed that the experimental lists would be longer than the practice lists, but were not informed of list length or number of trials.

A typical trial commenced with a warning signal (visual presentation of "READY"), followed by 16 to-be-remembered pictures or words, followed by a recall or track signal. The stimuli were presented at a $1.5 \mathrm{sec} / \mathrm{item}$ rate (onset to onset). The experimenter interrupted stimulus presentation and simultaneously started a hand-held stopwatch when a track or recall signal appeared. A $60-\mathrm{sec}$ recal period followed. The recall or track signal was projected for the duration of the recall period. (For Study 2, the equipment was wired such that interrupting stimulus presentation simultaneously activated a Flexopulse interval timer that timed the recall period.) The experimenter ended a trial by saying "stop." Subjects were instructed to discontinue recall and tracking upon hearing "stop."

Materials. The stimuli were slides of black-and-white line drawings of common objects, foods, and animals, or their corresponding verbal labels typed in black capital letters. The slides were rear projected via Kodak Carousel projector. Presentation rate was governed by a Flexopulse interval timer. A Lafayette shutter was placed on the projector lens and the aperture adjusted to reduce brightness and aftereffects. Subjects were seated at a table. A Lafayette pursuit-rotor tracker was in front of the subjects and rotated at 60 revolutions $/ \mathrm{min}$ throughout the experimental session. The rear-projection screen was immediately behind the pursuit-rotor tracker and obstructed the subjects' view of the remaining equipment and the experimenter. The stylus and target of the pursuit-rotor tracker were connected to a single Hunter Klockounter $(120 \mathrm{~A})$ so that time-on-target could be recorded. (For Study 2, the stylus and target were connected to two Klockounters via a relay. The relay was controlled via a second Flexopulse interval timer so that time-on-target was registered on a separate Klockounter for each 30-sec period.) Subjects held the stylus in their dominant hand throughout the experiment. Recall protocols were recorded via tape recorder.

\section{Results}

Recall. The picture-recall data were scored for both conservative and liberal criteria. For the conservative criterion, a response was scored correct if the corresponding label, form of the label (e.g., phone for telephone), or synonym was recalled. For the liberal criterion, a response was scored correct if the recalled label was a reasonable match with a picture in the pertinent list (e.g., mug for cup, parakeet for parrot, etc.). The word-recall data were scored in the same manner. The data were subjected to a 2 (replications) by 4 (order of conditions) by 2 (presentation mode) by 2 (recall activity) mixed analysis of variance. There were no substantial differences in results for conservative and liberal scoring. The results for liberal scoring are reported.

The analysis showed no significant main effects for either replications or order of conditions (ps $>.10$ ). Likewise, there were no significant interactions involving these between-subjects factors and the within-subjects factors of presentation mode and recall activity (all ps $>.10$ ). Pictures were not recalled better than words (mean items recalled were 7.57 and 7.54 , respectively, $\mathrm{p}>.10$ ), but more items were recalled in the control condition than in the tracking condition [mean items recalled were 7.92 and 7.20 , respectively, $F(1,24)=8.24$, MSe $=2.00, p<.01]$. Table 1 shows the significant interaction of Presentation Mode by Recall Activity $[\mathrm{F}(1,24)=8.47, \mathrm{MSe}=3.77, \mathrm{p}<.01]$.

Subjects recalled approximately two fewer pictures when tracking, but approximately the same number of words when tracking. The PC $>$ PT difference was reliable across subjects: Twenty-one showed PC $>$ PT, six showed PC $=$ PT, and five showed $\mathrm{PC}<\mathrm{PT}$ (Sign test, $\mathrm{z}=2.68, \mathrm{p}<.01)$. The WC $<\mathrm{WT}$ difference was not significant $(p>.10)$. 
Table 1

Means of Number of Items Recalled as a Function of Presentation Mode and Recall Activity

\begin{tabular}{ccc} 
& \multicolumn{2}{c}{ Recall Activity } \\
\cline { 2 - 3 } Presentation & Control & Track \\
\hline Mode & 8.43 & 6.71 \\
Picture & 7.40 & 7.68 \\
Word & & \\
\hline
\end{tabular}

Time-on-target. Time-on-target was recorded for each subject in the WT and PT conditions. The means for the WT and PT conditions were $18.98 \mathrm{sec}$ and $18.91 \mathrm{sec}$, respectively. These means were not significantly different $(p>.10)$.

\section{Discussion}

The results were quite clear in showing that recallconcurrent pursuit-rotor tracking interfered with the recall of pictures, but not with the recall of corresponding picture labels. The time-on-target data suggested that the effect was not simply due to subjects' spending more time tracking in the PT condition than in the WT condition. Furthermore, since the tracking conditions were compared with appropriate control conditions (see Clayton \& Warren, 1976), the results indicated that recall-concurrent pursuit-rotor tracking produced modality-specific interference for picture recall.

It is notable that the interaction of Presentation Mode by Recall Activity is consistent with the dualcoding model. According to the dual-coding model, the degree of availability of the imaginal code varies as a function of stimulus concreteness. Thus, the imaginal code is most available to pictures, somewhat less available to concrete words, and unavailable to abstract words (Paivio, 1971). On this basis, one would expect a large imaginal component in picture retrieval, a somewhat smaller imaginal component in concrete-word retrieval, and no imaginal component in abstract-word retrieval. Likewise, if it is assumed that two independent working memory systems, visual working memory and verbal working memory, mediate retrieval for the imaginal and verbal codes, then one would expect a visual recall-concurrent task to capture some proportion of the visual working memory processing capacity normally allocated to imaginal retrieval, thereby interfering with picture recall. Of course, this explanation requires a visual recall-concurrent task also to interfere with concrete-word recall. However, the degree of interference should vary with the likelihood of imaginal coding. Thus, the failure to observe an effect of tracking on concrete-word recall in this experiment could be attributed to the rapid presentation rate's precluding imaginal coding of concrete labels (see Paivio, 1975, p. 71). Consequently, all aspects of the observed interaction of Presentation Mode by Recall Activity can be accommodated by the dual-coding model.
The interaction of Presentation Mode by Recall Activity is somewhat more difficult to reconcile with a uniprocess explanation of picture and word recall. There are several reasonable criteria which constrain any uniprocess explanation of picture and word recall: (1) Words and pictures are assumed to be encoded and represented in the same manner; (2) pictures and words are assumed to differ on some dimension of encodability or retrievability to explain the picture-superiority effect; (3) retrieval is assumed to be mediated by a single process. However, these criteria do not prevent a post hoc explanation of the interaction of Presentation Mode by Recall Activity.

The uniprocess explanation would suggest that, although pictures are better or more likely to be encoded than are words, they require more working memory capacity for retrieval. Thus, a recall-concurrent distractor task would be more likely to capture processing capacity during picture retrieval, thereby prompting more interference and poorer recall. Furthermore, if it is assumed that not all available processing capacity is normally allocated to retrieval and recall, then the absence of an effect of tracking on word recall can be attributed to the distractor task's capturing "leftover" processing capacity.

It is important to note that the uniprocess explanation rests on the assumption that picture retrieval requires more processing capacity than does word retrieval. Without this assumption, both word and picture recall would be equally susceptible to a recallconcurrent distractor task. Neither this assumption nor a rationale for the assumption are currently incorporated in the multiple-cues, active-encoding, or depthof-processing explanation of the picture-superiority effect. Thus, these explanations would require substantial revision in order to accommodate the interaction of Presentation Mode by Recall Activity. On the other hand, the dual-coding model can accommodate both the picture-superiority effect and the interaction of Presentation Mode by Recall Activity without revision. On this basis, it seemed appropriate to examine mode-specific explanations of tracking interference in the next experiment.

\section{EXPERIMENT 2}

Experiment 2 was intended to examine further the effect of recall-concurrent tracking on picture and word recall. One question of interest was whether items from the primacy-asymptote and recency portions of the picture serial-position curve would be differentially susceptible to tracking interference. It was considered likely that recently presented items might be more accessible, require less processing capacity for retrieval, and be less susceptible to tracking interference than early items. That is, one might expect recency items to be maintained in primary memory, so that they could 
be retrieved immediately and without the necessity of devoting processing capacity to memory "search." Thus, the retrieval of recency items might require less processing capacity than the retrieval of primacyasymptote items. This reasoning prompted the examination of serial-position effects.

Two mode-specific explanations for the effect of recall-concurrent tracking on picture recall were examined in Experiment 2: (1) Recall-concurrent tracking might slow the rate of picture retrieval without otherwise affecting accessiblity; and (2) recallconcurrent tracking might alter the criterion for picture recall. Consider the first hypothesis. If tracking merely slows the rate of picture retrieval without otherwise affecting accessibility, then it follows that an increase in the duration of the recall period should enable subjects to recall more PT items and raise their PT performance to the level of PC performance. For this result to obtain, $P T$ recall must continue after $P C$ recall has ended in order to offset the PC $>$ PT difference observed for $1-\mathrm{min}$ recall. In short, this hypothesis predicts that an analysis of the likelihood of recall over time should show PT recall starting at a lower level than PC recall, but declining at a slower rate. Consequently, the recall period of Experiment 2 was extended to $2 \mathrm{~min}$ and the rate of decline (items recalled per $30 \mathrm{sec}$ ) was examined.

An analysis of intrusion-error data might provide data relevant to the second hypothesis. If we consider recall as a signal-detection process (see Kintsch, 1970) of differentiating to-be-remembered items from previously presented and extraexperimental items, then it follows that a strict criterion would yield lower recall than a liberal criterion. This would explain the interaction observed in Experiment 1, by suggesting that recall-concurrent tracking produced a liberal-toconservative criterion shift for picture recall, but not for word recall. Such a criterion shift would necessarily entail a concurrent reduction in intrusion errors. Thus, the criterion-shift explanation predicts similar interactions of Presentation Mode by Recall Activity for the number of items recalled and the number of intrusion errors.

\section{Method}

Subjects. The subjects were 64 Vanderbilt University undergraduates participating to fulfill a requirement for introductory psychology. Eleven subjects were replaced due to equipment failure or failure to follow instructions.

Design and procedure. The design was a mixed factorial with one between-subjects factor and three within-subjects factors. The between-subjects factor was order of conditions. The within-subjects factors were presentation mode (P vs W), recall activity (C vs T), and serial position (1-16).

Subjects received one list in each of four conditions. The order of conditions (WC, WT, PT, PR; WT, PR, WR, PT; PR, PT, WT, WR; PT, WR, PR, WT) and the order of lists $(1,2,4,3$; $2,3,1,4 ; 3,4,2,1 ; 4,1,3,2)$ were counterbalanced via a pseudorandom Latin square. The four order of conditions were mapped on the four orders of lists, yielding 16 order- of-conditions by order-of-lists combinations. Thus, across a block of 16 subjects, each list appeared in each condition and ordinal position equally often. Each block of 16 subjects was randomly assigned to the 16 order-of-conditions by order-of-lists combinations. Serial position was randomized with the constraint that each item of a 16-item list appear equally of ten in each serial position for each condition, thereby requiring 16 random orders. Since each list appeared in each condition four times (each of four ordinal positions) for a block of 16 subjects, four blocks or 64 subjects were required to counterbalance serial position.

As in Experiment 1 (Study 2), the pursuit-rotor tracker was gated to two Hunter Klockounters via a relay that was controlled by a Flexopulse interval timer. The interval timer switched the relay every $30 \mathrm{sec}$ so that time-on-target for consecutive 30 -sec periods was recorded on separate Klockounters. The switching of the relay was accompanied by an audible click. The clicks were recorded coincidental with recall protocols and facilitated the scoring of recall by 30 -sec periods. With the exception of a 2-min recall period, the remaining experimental procedures were identical to those of Experiment 1 (Study 2).

Materials. The materials were identical to those of Experiment 1 (Study 2), with the exception that new lists were randomly generated from the same stimulus pool.

\section{Results}

Recall. The free recall data were scored for both liberal and conservative criteria and subjected to several analyses. The only substantial difference in the results for liberal and conservative scoring was that conservative scoring lowered the overall level of picture recall. The results for liberal scoring are reported.

An initial 4 (order of conditions) by 2 (presentation mode) by 2 (recall activity) analysis of the 2 -min recall data disclosed results similar to those of Experiment 1. The analysis showed no main effect for the between. subjects factor of order of conditions, nor were there any significant interactions of order of conditions with the within-subjects factors (all ps $>.10$ ). A main effect of presentation mode $[\mathrm{F}(1,60)=9.73, \mathrm{MSe}=3.54$, $\mathrm{p}<.01]$ showed that pictures were recalled better than words (mean items recalled were 9.07 and 8.34, respectively), and a main effect of recall activity $[F(1,60)=12.23, \mathrm{MSe}=2.36, \mathrm{p}<.001]$ showed better recall for the control condition relative to the tracking condition (mean items recalled were 9.04 and 8.37, respectively). The interaction of Presentation Mode by Recall Activity was not significant $(p>.10)$. However, the results of Experiment 1 provided the basis for the test of two a priori hypotheses concerning a simple main effect of tracking for picture recall (i.e., $\mathrm{WC}=\mathrm{WT}$ and $\mathrm{PC}>\mathrm{PT}$ ). $\mathrm{T}$ tests revealed that the

Table 2

Means of Number of Items Recalled as a Function of Presentation Mode and Recall Activity

\begin{tabular}{ccc}
\hline \multirow{2}{*}{$\begin{array}{c}\text { Presentation } \\
\text { Mode }\end{array}$} & \multicolumn{2}{c}{ Recall Activity } \\
\cline { 2 - 3 } & Control & Track \\
\hline Picture & 9.53 & 8.67 \\
Word & 8.65 & 8.12 \\
\hline
\end{tabular}


WC $>$ WT difference was not significant $(p>.10)$, while the PC $>$ PT difference was highly significant $[t(1,63)=3.61, p<.001]$. See Table 2 for the pertinent means. Indeed, the PC $>$ PT difference was reliable across subjects: Thirty-seven showed PC $>$ PT, 16 showed $\mathrm{PC}<\mathrm{PT}$, and 11 showed $\mathrm{PC}=\mathrm{PT}$ (Sign test, $\mathrm{z}=2.74, \mathrm{p}<.01)$. Thus, the a priori test for a simple main effect provided results consistent with the interaction of Presentation Mode by Recall Activity observed in Experiment 1.

In order to examine serial-position effects, the 2 -min recall data were further subjected to a 4 (order of conditions) by 2 (presentation mode) by 2 (recall activity) by 16 (serial position) mixed analysis of variance. Evidence that the primacy-asymptote and recency items were differentially affected by tracking would be revealed as an interaction of Presentation Mode by Recall Activity by Serial Position. The results showed a significant main effect of serial position $[\mathrm{F}(15,900)=13.18, \mathrm{MSe}=.25, \mathrm{p}<.001]$; however, the critical three-way interaction of Presentation Mode by Recall Activity by Serial Position was not significant $(\mathrm{p}>.10)$. Indeed, there were no significant interactions of any factor with serial position (all ps $>.10$ ). The remaining results were consistent with the initial analysis.

The slow-down hypothesis suggests that PT recall performance will equal PC recall performance if sufficient time is allowed for recall. One implication of this hypothesis is that the decline in the likelihood of recall over time should be slower for the PT condition than for the PC condition. Consequently, recall was scored by $30-\mathrm{sec}$ periods $(0-30,30-60,60-90$, and 90-120 sec) and subjected to a 4 (order of conditions) by 4 (recall periods) by 2 (presentation mode) by 2 (recall activity) by 16 (serial position) mixed analysis of variance. Any significant difference in the decline of the likelihood of recall over time for Conditions PC and PT would be expected to appear as a threeway interaction of Recall Period by Presentation Mode by Recall Activity. The critical interaction was not significant $(p>.10)$. Moreover, the main effect of recall period $[\mathrm{F}(3,180)=925.75, \mathrm{MSe}=.16$, $\mathrm{p}<.001]$ disclosed a rapid decline in the proportion of items recalled: $.42(0-30 \mathrm{sec}), .07(30-60 \mathrm{sec})$, $.02(60-90 \mathrm{sec})$, and $.01(90-120 \mathrm{sec})$. Two significant interactions: (1) Recall Period by Presentation Mode $[F(3,180)=2.99$, MSe $=.08, p<.05]$, and (2) Recall Period by Serial Position $[F(45,2700)=12.60$, MSe $=.09, \mathrm{p}<.001]$ were not particularly important for our purposes and will not be considered further.

Intrusion errors. Intrusion errors were analyzed by a 2 (presentation mode) by 2 (recall activity) withinsubjects analysis of variance. The results showed significant main effects of presentation mode $[F(1,63)=4.62$, $\mathrm{MSe}=.92, \mathrm{p}<.05]$ and recall activity $[\mathrm{F}(1,63)=4.89$, $\mathrm{MSe}=1.09, \mathrm{p}<.051 ;$ however, the interaction of Presentation Mode by Recall Activity was not significant
Table 3

Means of Number of Intrusions as a Function of Presentation Mode and Recall Activity

\begin{tabular}{ccc} 
Presentation & \multicolumn{2}{c}{ Recall Activity } \\
\cline { 2 - 3 } Mode & Control & Track \\
\hline Picture & .75 & .65 \\
Word & 1.20 & .71 \\
\hline
\end{tabular}

$(p>.10)$. Significantly more intrusions were observed for word presentation relative to picture presentation (mean number of intrusions were .96 and .70 , respectively), and significantly fewer intrusions were observed for recall-concurrent tracking relative to the control condition (mean number of intrusions were .68 and .97 , respectively).

Consistent with the initial recall analysis, $t$ tests were used to test two a priori hypotheses concerning a simple main effect of tracking for picture-presentation intrusions (i.e., WC $=W T$ and PC $>$ PT). These tests revealed that the WC $>$ WT difference was significant $[t(1,63)=2.09, p<.05]$, while the PC $>$ PT difference was not significant $(p>.10)$. The pertinent means appear in Table 3.

Time-on-target. Time-on-target was recorded separately for each of the four 30-sec tracking periods comprising the 2-min recall period. Three missing scores were replaced by the mean for the remaining three 30 -sec periods. Times were summed to provide a single measure of time-on-target for the WT and PT conditions. The means for the WT and PT conditions were 43.34 and $42.73 \mathrm{sec}$, respectively. A t test showed that these means were not significantly different $(p>.10)$. Thus, there was no evidence of a difference in tracking performance for the WT and PT conditions.

\section{Discussion}

The results of Experiment 2 provided additional evidence that recall-concurrent pursuit-rotor tracking selectively interferes with picture recall. The a priori tests for a simple main effect of tracking on picture serial recall disclosed a significant PC $>$ PT difference and a nonsignificant $W C>W T$ difference. The failure to observe a significant interaction of Presentation Mode by Recall Activity was somewhat perplexing, though, because the experimental procedures and materials in Experiments 1 and 2 were very similar. Consequently, it was not obvious what change in Experiment 2 accounted for the absence of the interaction of Presentation Mode by Recall Activity. However, if one examines the overall pattern of results from Experiments 1 and 2, the results are quite reliable. A test of the PC-PT difference for the three independent experiments showed a significant $\mathrm{PC}>\mathrm{PT}$ difference for all three experiments: (1) Experiment 1, Study 1 $[\mathrm{t}(1,16)=3.87, \mathrm{p}<.01]$, (2) Experiment 1, Study 2 $[t(1,16)=2.12, \quad p<.05], \quad$ and (3) Experiment 2 $[t(1,63)=3.61, p<.001]$. However, a test of the 
WC-WT difference for the three independent experiments failed to disclose a single significant WC $>$ WT difference: (1) Experiment 1, Study 1, WC $<$ WT $[\mathrm{t}(1,16)=1.04, \mathrm{p}>.10]$, (2) Experiment 1, Study 2, $\mathrm{WC}=\mathrm{WT}[\mathrm{t}(1,16)=.00, \mathrm{p}>.10]$, and (3) Experiment 2, $W C>W T \quad[t(1,63)=1.53, p>.10]$. Thus, in three experiments, recall-concurrent tracking produced a significant decline in picture recall, but not in word recall. There was only one instance in which recallconcurrent tracking reduced word recall, and the reduction was not statistically significant.

The serial-position analysis provided no support for the hypothesis that primacy-asymptote and recency items might differ in their susceptibility to recallconcurrent tracking. The results showed that tracking reduced recall over the entire serial-position curve. Thus, it appeared that the retrieval of primacyasymptote and recency items required roughly the same amount of processing capacity.

The examination of the likelihood of recall over successive 30-sec recall periods provided no support for the slow-down hypothesis. The hypothesis predicts that an analysis of the likelihood of recall over time will show a slower decline in recall for the PT condition relative to the $\mathrm{PC}$ condition. The prediction was not supported.

The criterion-shift hypothesis predicted matching results for the recall and intrusion-error analyses. The results for picture presentation did not satisfy this requirement; a significant $\mathrm{PC}>\mathrm{PT}$ difference was observed for recall, but was accompanied by a nonsignificant PC $>$ PT difference in intrusions. Moreover, a nonsignificant WC $>$ WT recall difference was accompanied by a significant WC $>$ WT difference in intrusions. Thus, the overall pattern of results suggested that recall-concurrent tracking generally suppressed the willingness to respond; however, the overall pattern of results was not consistent with the hypothesis that tracking produced a selective criterion shift for picture recall.

Thus, the results of Experiment 2 were not particularly informative. The results failed to support the slowdown and criterion-shift explanations of the selective effect of recall-concurrent tracking on picture recall. However, the results did provide further support for the suggestion that picture recall entails a larger imaginal component than does word recall.

\section{EXPERIMENT 3}

The results of the previous experiments were consistent with the dual-coding model's prediction of a larger imaginal component for picture recall relative to word recall. A further test of the dual-coding model would be provided by an examination of the effect of tracking on concrete and abstract word recall. Remember, according to the dual-coding model, the imaginal code is most available to pictures, somewhat less available to concrete words, and unavailable to abstract words (Paivio, 1971). Thus, the dual-coding model predicts a larger imaginal component for the recall of concrete words relative to abstract words. To test this prediction, subjects in Experiment 3 were presented concrete and abstract word lists and required to recall or to recall while tracking. Likewise, a slower presentation rate was used to encourage imaginal coding of the concrete items.

\section{Method}

Subjects. The subjects were 28 Vanderbilt University undergraduates participating to fulfill a requirement for introductory psychology. Two subjects were eliminated due to equipment failure or failure to follow instructions.

Design and procedure. The design was a completely withinsubjects factorial, comparing type of list (concrete, $C$, vs abstract, A), recall activity (control, $\mathrm{C}$, vs tracking, $\mathrm{T}$ ), and trials (three lists in each of four conditions). Each subject received a different random ordering of the 12 conditions-by-trials lists and a different random ordering of the words within each list. The order of lists and words within lists was determined by FORTRAN algorithm. Lists were counterbalanced with conditions, so that all the concrete and abstract lists served in the control and track conditions equally of ten.

Subjects were informed that the experiment was intended to examine the relationship between memory and motor performance. Instructions for the control and track conditions were identical to those of Experiments 1 and 2. Subjects were informed that they would have $1 \mathrm{~min}$ for free oral recall, were encouraged to guess, and were told not to be afraid to repeat items. Subjects were not informed of list length, number of trials, or the manipulation of item concreteness. Two practice trials (control trial and track trial) on the same 16-item mixed list (eight $C$ and eight $A$ items) served to familiarize the subjects with the experimental procedure. The stimuli were rear projected at a 5 -sec/item rate (onset to onset). A warning slide ("XXXX") preceded each list and a recall ("*ECALL") or track ("*TRACK*") slide terminated each list. The remaining procedures were identical to those of Experiment 1 (Study 2).

Materials. The stimulus words were selected via FORTRAN algorithm from the Paivio, Yuille, and Madigan (1968) norms. The algorithm selected $\mathrm{A}$ words with imagery and concreteness ratings of 5.00 or less, meaningfulness ratings of 4.00 or higher, and Thorndike-Lorge frequencies of 35 per million or higher (words with AA and A frequencies were assigned values of 99 and 50 per million). The algorithm selected $C$ words with imagery and concreteness ratings of 6.00 or higher, meaningfulness ratings of greater than 4.00 but less than 7.20 , and Thorndike-Lorge frequencies of 35 per million or higher. The algorithm yielded an A pool and a C pool of 98 and 99 words, respectively. A second FORTRAN algorithm randomly generated six lists of 16 items from the respective pools. The $A$ and $C$ lists were approximately equated on meaningfulness (mean rating for $\mathrm{A}$ lists, 5.64; mean rating for $\mathrm{C}$ lists, 6.36) and Thorndike-Lorge frequency (mean rating for $A$ lists, 70.98; mean rating for $C$ lists, 74.38). However, the $C$ lists exceeded the $A$ lists in mean rated imagery $(6.40$ vs 3.62$)$ and mean rated concreteness (6.75 vs 2.68 ).

The words were typed in black uppercase block letters, Xeroxed on Nashua Xerographic transparency film, and mounted in Kodak slides. The remaining materials were identical to those in Experiment 1 (Study 2).

\section{Results}

Recall. The free recall data were subjected to a 3 (trials) by 2 (type of list) by 2 (recall activity) withinsubjects analysis of variance. The analysis revealed a significant main effect of type of lists $[F(1,27)=136.85$, 
Table 4

Means of Number of Items Recalled as a Function of Type of List and Recall Activity

\begin{tabular}{ccr} 
& \multicolumn{2}{c}{ Recall Activity } \\
\cline { 2 - 3 } Presentation & Control & Track \\
\hline Mode & 11.10 & 10.81 \\
Concrete & 8.03 & 8.30 \\
\hline
\end{tabular}

$\mathrm{MSe}=4.76, p<.001]$. None of the remaining main effects and interactions were significant (all ps $>.10$ ). Thus $\mathrm{C}$ lists were better recalled than $\mathrm{A}$ lists (mean items recalled were 10.95 and 8.17 , respectively), but there was no significant difference in recall across trials (mean items recalled were $9.75,9.29,9.69$ ) or between the control and tracking conditions (mean items recalled were 9.57 and 9.55 , respectively).

An additional analysis examined the a priori hypothesis of a simple main effect of tracking for concrete-list recall (i.e., $\mathrm{AC}=\mathrm{AT}$ and $\mathrm{CC}>\mathrm{CT}$ ). Table 4 shows the pertinent means. Comparison of mean recall scores, collapsed across trials, revealed that neither the AC $<$ AT difference, nor the $\mathrm{CC}>\mathrm{CT}$ difference was significant (ps $>.10)$. Thus, there was no evidence that recall-concurrent tracking interfered with concretelist recall.

Time-on-target. Time-on-target was recorded separately for the two $30-\mathrm{sec}$ periods comprising the 1 -min recall period. Three missing scores were replaced by the mean of the subjects' remaining trials. Times were subjected to a 3 (trials) by 2 (tracking condition: AT vs CT) within-subjects analysis of variance. The results showed that tracking performance improved over trials [mean time-on-target in seconds: 21.59, $25.87,28.50, \mathrm{~F}(2,54)=28.91, \mathrm{MSe}=23.54, \mathrm{p}<.001]$; however, there was no significant difference between the AT and CT conditions (mean in seconds: 25.14 and 25.47 , respectively, $F<1$ ). Thus, there was no evidence of a difference in tracking performance for the AT and CT conditions.

\section{Discussion}

The recall analysis disclosed that concrete lists were recalled significantly better than were abstract lists. This result replicates previous experiments (e.g., Paivio \& Csapo, 1969, 1973) in demonstrating the superior free recall of concrete stimuli, and, according to the dual-coding hypothesis, may be interpreted as evidence that the concrete stimuli received imaginal coding. The analysis failed to show a significant effect of recall-concurrent tracking on the recall of concrete lists. However, the failure to obtain an effect of tracking was clearly not due to inadequate differences in level of concreteness or to overlearning of concrete lists masking significant differences in strength, as there was a substantial difference in performance between the two types of lists and no evidence of a ceiling effect.
Thus, the results provided further empirical evidence that recall-concurrent tracking does not interfere with word recall; however, the results were not consistent with the dual-coding prediction.

Experiment 3 provided no evidence that the recall of concrete lists involved a larger imaginal component than did the recall of abstract lists. This result was rather surprising, as the superior recall of concrete over abstract lists is generally attributed to the increased availability of the imaginal code to concrete items (Paivio, 1971). Thus, the results suggested that the concrete items were afforded imaginal coding, yet the results showed no evidence of an imaginal component in the recall of the concrete items.

One implication of the results is that the difference in the recall of concrete and abstract lists may not reflect the imaginal coding of the concrete items. How then can the superior recall of concrete lists be explained? Baddeley et al. (1974) have provided a tentative explanation. They have suggested that concreteness might be "a semantic feature of a word whose effect on memory is not accessible to visual disruption." That is, the rated concreteness of a verbal stimulus is something other than an index of the likelihood of imaginal coding. Of course, a semanticfeature explanation of the results of Experiment 3 must be viewed with some skepticism because it rests on the acceptance of the null hypothesis with regard to the effect of tracking on concrete-list recall. On the other hand, Baddeley et al. have reported a similar result. Indeed, their semantic-feature explanation was based on their inability to demonstrate an interaction between the concreteness of noun-adjective pairs (concrete vs abstract) and presentation-concurrent pursuit-rotor tracking (present vs absent).

An alternative implication of the results is that the imagery system that mediates picture recall may differ in some important way from the imagery system mediating concrete-word recall (cf. Janssen, 1976). Concrete words might be stored in an imaginal format that renders them less susceptible to the visual interference produced by recall-concurrent pursuit-rotor tracking. This explanation preserves the dual-coding of concrete words, but it constitutes a significant revision of the dual-coding model. That is, the explanation requires that concrete words and pictures differ not only in the degree of imaginal coding, but also in the quality or type of imaginal coding. Thus, both the present explanation and the semantic-feature explanation of the results of Experiment 3 suggest a revision of the dual-coding model.

\section{CONCLUSIONS}

Experiments 1 and 2 were consistent with the dualcoding prediction of a larger imaginal component in the recall of pictures relative to words: Recall-concurrent 
pursuit-rotor tracking interfered with the recall of pictures, but not of words. Current uniprocess explanations of the picture-superiority effect would require substantial revision to accommodate the results. Experiment 3 failed to show an effect of tracking on the recall of concrete lists, in spite of a substantial difference in the recall of concrete and abstract lists. This result was inconsistent with the dual-coding model's prediction and suggested a need for revising the dual-coding model.

\section{REFERENCE NOTES}

1. Pellegrino, J. W., Rosinski, R. R., \& Siegel, A. W. Picture-word differences in semantic decision latency: An analysis of single and dual-memory models. Paper presented at a meeting of the Psychonomic Society, 1975.

2. Brooks, L. R. Visual and verbal processes in internal representation. Paper presented at the Salk Institute, La Jolla, California, July 1970.

\section{REFERENCES}

Baddeley, A. D., Grant, S., Wight, E., \& Thomson, N. Imagery and visual working memory. In P. M. Rabbitt \& S. Dornic (Eds.), Attention and performance (Vol. 5). New York: Academic Press, 1974.

Bousfield, W. A., Esterson, J., \& Whitmarsh, G. A. The effects of colored and uncolored pictorial representations on the learning of stimulus words. Journal of Applied Psychology, 1957, 41, 165-168.

BYRNE, B. Item concreteness vs. spatial organization as predictors of visual imagery. Memory \& Cognition, 1974, 2, 53-59.

Clayton, K. N., \& Warren, M. W. Methodological problems with the use of the retroactive-interference design to infer what is stored. Memory \& Cognition, 1976, 4, 237-243.

Cohen, G. How are pictures registered in memory? Quarterly Journal of Experimental Psychology, 1973, 25, 557-564.

CRAIK, F. I. M., \& LockhART, R. S. Levels of processing: A framework for memory research. Joumal of Verbal Learning and Verbal Behavior, 1972, 11, 671-684.

Davies, G. M., Milne, J. E., \& Glennie, B. J. On the significance of "double encoding" for the superior recall of pictures t to names. Quarterly Journal of Experimental Psychology, 1973, 25, 413-423.

JANSSEN, W. H. Selective interference in paired-associate and free recall learning: Messing up the image. Acto Psychologica, 1976, 40, 35-48.

KINTSCH, W. Learning, memory, and conceptual processes. New York: John Wiley, 1970.

Lieberman, L. R., \& Culpepper, J. T. Concrete image and verbal memory codes. Psychological Reports, 1965, 17, 983-988.

MAdigaN, S. Representational storage in picture memory. Bulletin of the Psychonomic Society, 1974, 4, 567-568.

May, J. E., \& Clayton, K. N. Imaginal processes during the attempt to recall names. Journal of Verbal Learning and Verbal Behavior, 1973, 12, 683-688.

Nelson, T. O., Metzler, J., \& Reed, D. A. Role of details in the long-term recognition of pictures and verbal descriptions. Journal of Experimental Psychology, 1974, 102, 184-186.

PaIvio, A. Imagery and verbal processes. New York: Holt, Rinehart, \& Winston, 1971.

Parvio, A. Imagery and long-term memory. In R. A. Kennedy \& A. Wilkes (Eds.), Studies in long term memory. New York: John Wiley, 1975.

Paivio, A., \& Csapo, K. Concrete-image and verbal memory codes. Joumal of Experimental Psychology, 1969, 80, 279-285.

Parvio, A., \& Csapo, K. Picture superiority in free recall: Imagery or dual coding? Cognitive Psychology, 1973, 5, 176-206.

Paivio, A., Rogers, T. B., \& Smythe, P. C. Why are pictures easier to recall than words? Psychonomic Science, $1968,11,137-138$.

Paivio, A., Yuille, J., \& Madigan, S. Concreteness, imagery and meaningfulness values for 925 nouns. Journal of Experimental Psychology Monograph Supplement, 1968, 76(1, part 2).

Pellegrino, J. W., Siegel, A. W., \& Dhawan, M. Shortterm retention of pictures and words: Evidence for dual coding systems. Journal of Experimental Psychology: Human Learning and Memory, 1975, 104, 95-102.

Pellegrino, J. W., Siegel, A. W., \& Dhawan, M. Shortterm retention of pictures and words as a function of type of distraction and length of delay interval. Memony \& Cognition, 1976, 4, 11-15.

SAMPSon, J. R. Free recall of verbal and nonverbal stimuli. Quarterly Joumal of Experimental Psychology, 1970, 22, 215-221.

Scotr, K. G. Clustering with perceptual and symbolic stimuli in free recall. Journal of Verbal Learning and Verbal Behavior, 1967, 6, 864-866.

SEgal, S. J., \& Fusella, V. Influence of imaged pictures and sounds on detection of visual and auditory signals. Journal of Experimental Psychology, 1970, 83, 458-464.

Wells, E. J. Words and pictures as distinct encoding categories in short-term memory. Journal of Experimental Psychology, 1973, 97, 394-396.

WiCker, F. W., \& Holley, F. M. Distraction modality and stimulus modality in paired-associate learning. Psychonomic Science, 1971, 25, 218-220.

(Received for publication July 27, 1976; revision accepted December 29, 1976.) 